\title{
Bilateral linear lichen planus along the lines of Blaschko: Report of a rare case and brief review
}

\author{
Mohammadreza Mortazavi', Somaia Ahmed Saad El-Din ${ }^{2}$
}

\author{
${ }^{1}$ Department of Dermatology, Hayat International Hospital, Muscat, Oman, ${ }^{2}$ Department of Pathology, Faculty of Medicine, \\ Ain Shams University, Cairo, Egypt
}

Corresponding author: Dr. Mohammadreza Mortazavi, E-mail: mmortazavir@gmail.com

\begin{abstract}
Linear lichen planus (LP) is a rare variant of lichen planus that may present in a dermatomal or zosteriform distribution or develop along the lines of Blaschko. Blaschkoid linear LP is a rarer distinct variant of LP and should be differentiated from other linear subtypes of LP and other Blaschkoid dermatoses. A 24-year-old male patient presented with multiple shiny, purple, polygonal papules arranged perpendicularly over the right lower extremity and also in shorter curvilinear (S-shaped) lesions on the left side of the abdomen and left flank along the lines of Blaschko. The histopathological findings of a biopsy specimen were compatible with the clinical diagnosis of lichen planus. To date, almost all of the reported cases of linear lichen planus along the lines of Blaschko have had unilateral presentations. We herein report a rare case of bilateral linear Blaschkoid lichen planus and review the literature briefly.
\end{abstract}

Key words: Lichen planus; Linear lichen planus; Blaschkoid lichen planus; Bilateral

\section{INTRODUCTION}

Lichen planus (LP) is a common cutaneous disorder that usually presents as pruritic, purple or violaceous, polygonal, planar (flat-topped) papular skin lesions, but it may also affect the mucous membranes, nails and hairs. The clinical presentation of lichen planus includes several different morphological variants such as hypertrophic, atrophic, actinic, pigmented (LP pigmentosus), annular, follicular (lichenplanopilaris), and linear LP [1]. Linear LP may arise in a dermatomal or zosteriform distribution (i.e. following the course of a peripheral nerve) either de novo or at the sites of trauma (isomorphic response or Koebnerization) [2] or as Wolf's isotopic response at the site of healed herpes zoster [3]. In addition, linear LP may present with development of lesions along the lines of Blaschko [3,4]. Blaschko's lines do not correspond to the nervous, vascular or lymphatic structures and represent a form of mosaicism that develops during the embryonic period [5]. Linear LP has been described in less than $1 \%$ of all LP patients [3]. Almost all of the reported cases of linear lichen planus along the lines of Blaschko presented unilaterally $[3-5,8-10]$. We herein report a rare case of bilateral linear Blaschkoid lichen planus.

\section{CASE REPORT}

A 24-year-old man presented with multiple itchy, purple lesions on the right lower extremity since five months ago. The lesions started from his right lower leg, gradually extended to the right thigh and buttock area in a linear manner and recently appeared on the left side of his abdomen. The patient had history of glucose6-phosphate dehydrogenase (G6PD) deficiency, migraine headaches and has been recently diagnosed with mild familial antithrombin III deficiency without any thrombotic event up to now. He had family history of serious and even fatal thromboembolism due to antithrombin III deficiency. After full medical investigations, he was advised to take prophylactic anticoagulants just for travel and major surgery. He

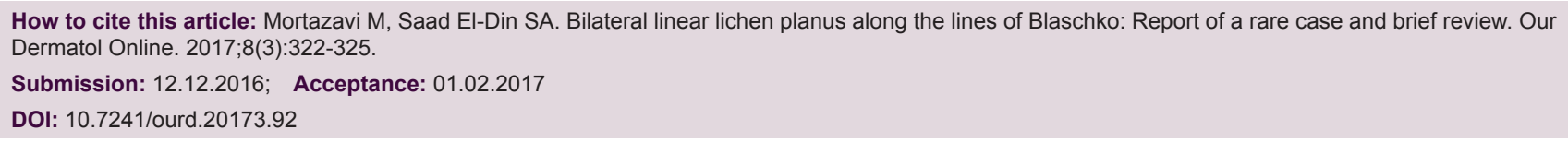


has not been on any regular medications during last 5 to 6 months, except occasional and random intake of NSAIDs (Ibuprofen \& Mefenamic acid), Paracetamol and Naratriptan for a sport left knee injury and for his migraine headaches.

The findings in general physical examination were unremarkable and the skin examination showed multiple shiny, purple, polygonal flat-topped papules distributed from the right lower leg to the medial and then lateral sides of the right thigh, up to the right buttock in a linear pattern along the lines of Blaschko (Fig. la and b). Also, there were a few shorter curvilinear (S-shaped) lesions consist of flat violaceous papules on the left side of the abdomen and left flank (Fig. lc). In addition, the arrangement of several similar micropapules in a short vertical line on the right elbow was noted as an isomorphic response to scratching (Koebner phenomenon) (Fig. ld). Other areas of the skin including scalp, nails, and also genital and oral mucosa were spared.

The results of routine laboratory tests including complete $\mathrm{CBC}$ and liver function tests were all within normal limits and the serology tests for hepatitis $\mathrm{B}$ and $\mathrm{C}$ were reported negative.

Punch biopsy of a papule on the right thigh was performed with the clinical diagnosis of linear Blaschkoid lichen planus and to rule out linear psoriasis, lichen striatus and other linear lesions. The specimen was stained with Hematoxylin \& Eosin (H \& E). The histopathological examination (Figs. 2a-c) revealed mild hyperkeratosis (orthokeratosis) with occasional foci of parakeratosis, prominent granular cell layer, foci of irregular acanthosis with sawtooth appearance of the rete ridges; dense band-like lymphocytic inflammatory infiltrate, obscuring the dermal-epidermal junction associated with basal cell vacuolization, apoptosis of the basal cells with formation of the characteristic eosinophilic Civatte (colloid) bodies. The lymphocytes in the infiltrate were intermingled with some macrophages, melanophages and eosinophils. These histopathological findings were compatible with the clinical diagnosis of linear lichen planus. Moreover, the absence of the features such as psoriasiform epidermal hyperplasia, thinning out of the supra-papillary epidermis, neutrophilic epidermal exocytosis, and deep reticular dermal perifollicular and perieccrine inflammatory infiltrate excluded other clinical differential diagnoses, i.e., linear psoriasis and lichen striatus, respectively.

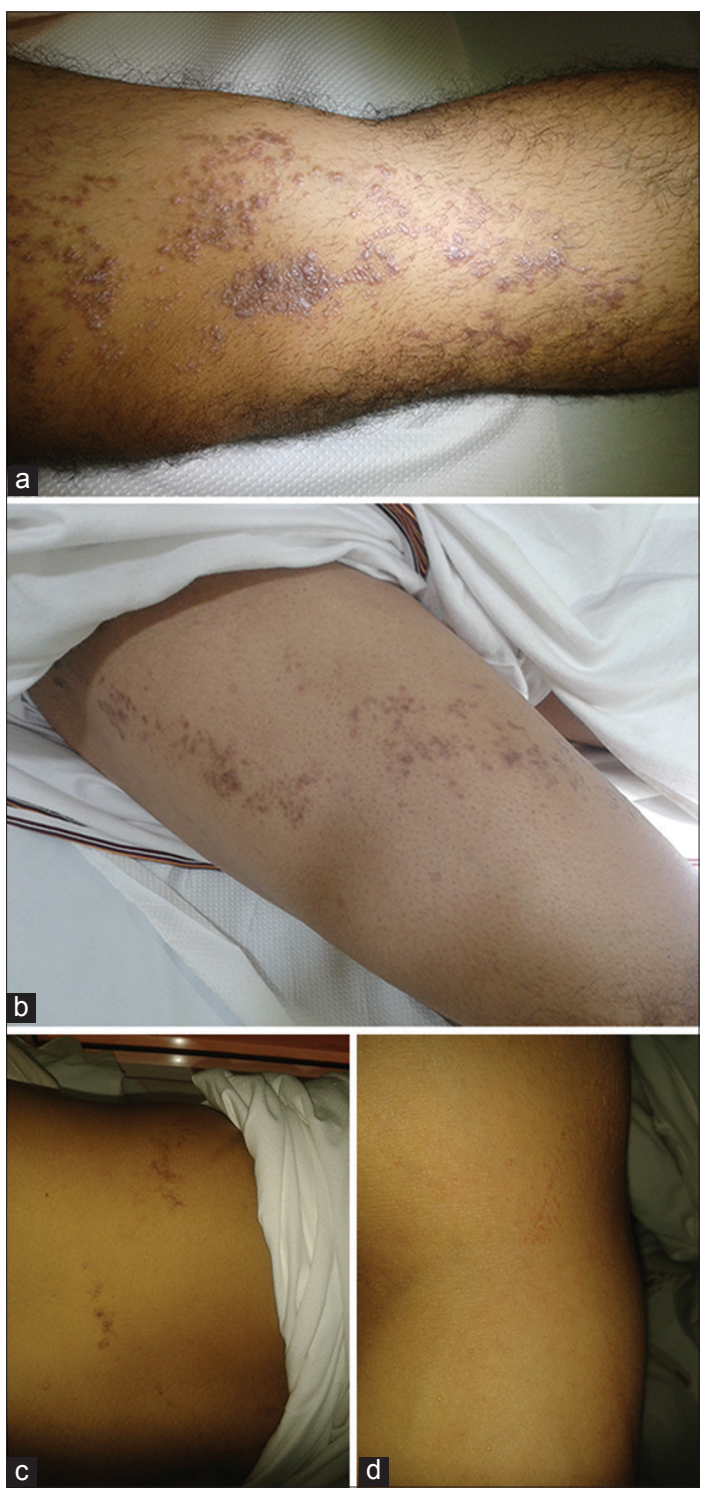

Figure 1: Clinical: ( $a$ and b) Shiny, purple, polygonal grouped papules distributed from the right lower leg to the medial and then lateral sides of the right thigh in a linear pattern along the lines of Blaschko; (c) flat, violaceous, grouped papules arranged in curvilinear (S-shaped) lesions on the left side of the abdomen and left flank; (d) a linear lesion consists of shiny micropapules on the right elbow.

With a final diagnosis of linear Blaschkoid lichen planus, treatment was started by oral antihistamines, topical Betamethasone valerate (Betnovate cream) and emollients. After 2 weeks, topical Betamethasone was replaced by topical Clobetasol propionate (Dermovate cream) to accelerate his improvement. The patient responded well to topical corticosteroid therapy.

\section{DISCUSSION}

Linear lichen planus along the lines of Blaschko is one of the rare morphological presentations of LP [5]. 


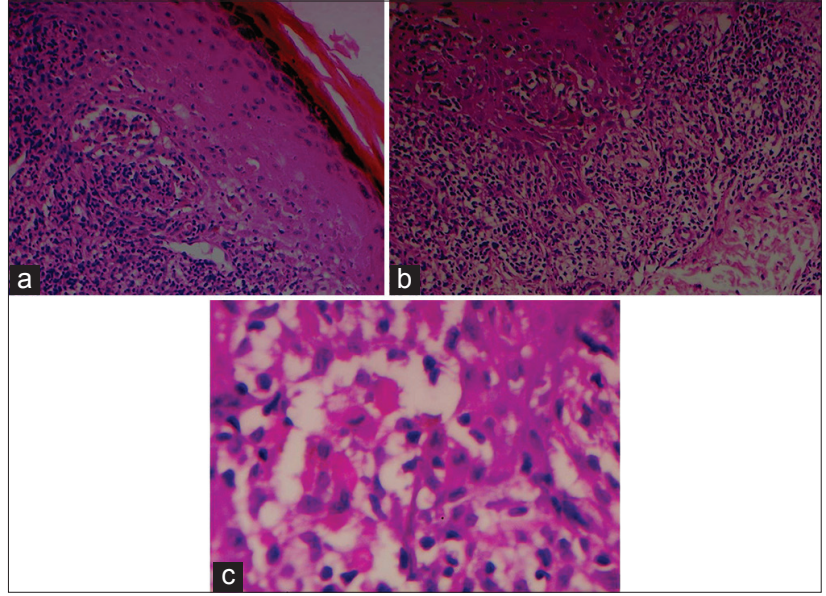

Figure 2: Histopathology: (a) Dense band-like inflammatory infiltrate obscuring the dermal-epidermal junction associated with mild hyperkeratosis, prominent granular cell layer, epidermal lymphocytic exocytosis and vacuolated basal cells, H\&E, x100; (b) Focal sawtoothed appearance of rete ridges surrounded by dense lymphocytic infiltrate H\&E, x100.; (c) Eosinophilic Civatte bodies (apoptotic basal cells) at the superficial dermis, H\&E, x400.

The lines of Blaschko were described in 1901 by the German dermatologist, Alfred Blaschko as the linear developmental patterns of epidermal and sebaceous nevi in more than 150 patients [6]. Jackson, after 75 years, provided a detailed review of the concept of Balschko lines [6]. The lines of Blaschko represent a classic pattern of mosaicism, arises from two or more genetically different cutaneous cell populations in an individual derived from a single zygote, that can be seen in a wide variety of not only congenital but also acquired skin disorders, including lichen planus $[3,7]$. It is hypothesized that the mosaicism may increase susceptibility of the skin in that specific area to develop a particular multifactorial cutaneous disease. Establishing the diagnosis of an inflammatory acquired skin disease that follows the Blaschko lines requires careful consideration of the history (age of onset, symptoms, natural course, etc.) as well as the clinical and histologic features [7].

A review of the reported linear LP cases in the literature reveals a controversy about the use of the terms zosteriform LP versus Blaschkoid (or Blaschkoian) LP. Some of the reported "zosteriform" LP cases need to be revised as Blaschkoid LP $[4,8]$. To solve this controversy, Blaschko lines should be clearly distinguished from other linear patterns such as lines of innervation of spinal nerves (dermatomes), Langer's lines, etc. [5,9]. Zosteriform (dermatomal) patterns consistently correspond to the areas that are innervated by the spinal nerves but Blaschko lines follow some distinct developmental patterns and are V-shape over the upper spine, $\mathrm{S}$-shape on the abdomen, inverted U-shape from the breast area onto the upper arm and perpendicular over the front and back of the lower extremities [10]. Another source of confusion is the role of Koebner phenomenon in development of linear LP lesions. The koebner phenomenon satisfactorily explains most cases with linear as well as the zosteriform distribution of LP because they have appeared either secondary to a known trauma or in the healed lesions of a preexisting disease such as herpes zoster $[2,3,9]$. The exact cause of the Blaschkoid pattern of LP has not yet been verified but there is no evidence to support the role of Koebner phenomenon in its etiology.

Most of the reported Blaschkoid LP cases (including our case) present with typical papular lesions, but there are rare reports of other variants of LP in the literature such as LP pigmentosus [11], lichenplanopilaris, atrophic LP [12], and drug-induced LP [13] with linear Blaschkoid pattern.

Lichen planus is generally a cell-mediated immune reaction of unknown cause. An association between lichen planus and hepatitis $\mathrm{C}$ virus infection, chronic active hepatitis, and primary biliary cirrhosis has been reported [14]. In one meta-analysis, 16\% of LP patients had hepatitis $\mathrm{C}$ infection [14]. To date, no systemic associations, including hepatitis $\mathrm{C}$ have been reported with Blaschkoid LP [9], while other variants of linear LP may show such associations [15]. In our reported case, serology tests for hepatitis $\mathrm{B}$ and $\mathrm{C}$ were negative.

Our reported case was recently diagnosed with asymptomatic hereditary antithrombin III deficiency and glucose-6-phosphate dehydrogenase (G6PD) deficiency. The authors believe that this is the first reported comorbidity of these medical conditions and Blaschkoid type of LP in the literature. Considering the very high prevalence of erythrocyte G6PD deficiency in Oman's male population (up to 25\%) [16], the concurrent presence of it and LP in our reported case seems to be merely a co-incidence.

Bilateral linear presentation of Blashkoid skin diseases other than LP, such as linear Grover's Disease [17], lichen striatus [18] and linear morphea [19] have rarely been reported in the literature. Almost all of the reported Blaschkoid LP cases had presented with unilateral lesions. To the best of our knowledge, this is the second reported case of bilateral Blaschkoid LP after the Long \& Finlay's case [20]. 


\section{Consent}

The evaluation and examination of the patient was conducted according to the Declaration of Helsinki principles.

\section{REFERENCES}

1. Hartl C, Steen KH, Wegner H, Seifert H, Bieber T. Unilateral linear lichen planus with mucous membrane involvement. Acta Derm Venereol (Stockh). 1999;79:145-6.

2. Turan E, Akay A, Yesilova Y, Türkçü G. A case of zosteriform lichen planus developing after extracorporeal shockwave lithotripsy. Dermatol Online J. 2012;18:9.

3. Kota RS, Jivani NB, Nair PA. Lichen planus: Zosteriform or along the lines of Blaschko? Pigment Int. 2016;3:43-5.

4. Kabbash C, Laude TA, Weinberg JM, Silverberg NB. Lichen planus in the lines of Blaschko. Pediatr Dermatol. 2002;19:541-5.

5. Rather AP, Hassan I. Blaschkoid lichen planus in an adult Kashmiri male: A rare presentation. Our Dermatol Online. 2013;4:179-82.

6. Jackson R. The lines of Blaschko: A review and reconsideration. Br J Dermatol. 1976;95:349-60.

7. Molho-Pessach V, Schaffer J. Blaschko lines and other patterns of cutaneous mosaicism. Clin Dermatol. 2011;29:205-25.

8. Taniguchi Y, Minamikawa M, Shimizu M, Ando K, Yamazaki S. Linear lichen planus mimicking creeping eruption. J Dermatol. 1993;20:118-21.

9. Tagra S, Talwar A, Rattan LW. Lines of blaschko. Indian J Dermatol Venereol Leprol. 2005;71:57-9.

10. Lade NR, Saoji V, Singh AL. Blaschkoid lichen planus: not a Koebner phenomenon. Dermatol Online J. 2013;19:17.

11. Relhan V, Sethi S, Garg Vijay K, Khurana N. Lichen planus pigmentosus in linear and zosteriform pattern along the lines of Blaschko. Dermatol Online J. 2015;21:11.

12. Anna Michalska-Bańkowska, Alina Skrzypek-Salamon and Anna Lis-Święty. Linear lichen planus along the lines of Blaschko in three adult women: gene-environment interactions. Clin Dysmorphol. 2015;24:166-9.

13. Muñoz MA, Pérez-Bernal AM, Camacho FM. Lichenoid drug eruption following the Blaschko lines. Dermatology. 1996;193:66-7.

14. Shengyuan L, Songpo Y, Wen W, Wenjing T, Haitao Z, Binyou W. Hepatitis $C$ virus and lichen planus: a reciprocal association determined by a meta-analysis. Arch Dermatol. 2009;145:1040-7.

15. Vachiramon V, Suchonwanit P, Thadanipon K. Bilateral Linear Lichen Planus Pigmentosus associated with hepatitis $\mathrm{C}$ virus infection. Case Rep Dermatol. 2010;2:169-72.

16. Al-Riyami A, Ebrahim GJ. Genetic Blood Disorders Survey in the Sultanate of Oman. J Trop Pediatr. 2003;49(Suppl 1):1-20.

17. Asahina A, Ishiko A, Saito I, Hasegawa K, Sawamura D, Nakano H. Grover's disease following multiple bilateral Blaschko lines: A rare clinical presentation with genetic and electron microscopic analyses. Dermatology. 2012;225:183-7.

18. Mittal R, Khaitan BK, Ramam M, Verma KK, Manchanda M. Multiple lichen striatus: An unusual presentation. Indian J Dermatol Venereol Leprol. 2001;67:204.

19. Weibel L, Harper J I. Linear morphea follows Blaschko's lines. Br J Dermatol. 2008;159:175- 81.

20. Long CC, Finlay AY. Multiple linear lichen planus in the lines of Blaschko. Br J Dermatol. 1996;135:275-6.

Copyright by Mohammadreza Mortazavi, et al. This is an open-access article distributed under the terms of the Creative Commons Attribution License, which permits unrestricted use, distribution, and reproduction in any medium, provided the original author and source are credited.

Source of Support: Nil, Conflict of Interest: None declared. 